\title{
ASYMPTOTIC SPECTRUM OF THE OPERATOR AND CHARACTERISTIC FUNCTION OPERATOR STURM-LIOUVILLE
}

\author{
FATIH DESTOVIĆ ${ }^{1}$, ISMET KALČO ${ }^{2}$ and NEDŽAD DUKIĆ 1 \\ ${ }^{1}$ Faculty of Natural Sciences \\ University of Sarajevo and Faculty Educations \\ Sarajevo \\ Bosnia and Herzegovina \\ e-mail: faith_d@msn.com \\ ${ }^{2}$ Polytechnic Faculty \\ University of Zenica \\ Zenica \\ Bosnia and Herzegovina
}

\begin{abstract}
In this paper, we study some operators type Sturm-Liouville with delay construction of solutions of the equation method of successive approximations, the formation of the characteristic features of operators and the asymptotic behaviour of the characteristic features.
\end{abstract}

\section{Introduction}

Spectral analysis is a modern mathematical theory which proved to be extremely effective in addressing one very broad class of problems in various scientific disciplines such as mathematics, mechanics, physics, 2010 Mathematics Subject Classification: 45XX.

Keywords and phrases: characteristic function, asymptotic zero, linear delay, potential.

Received January 11, 2016

(ㄷ) 2016 Scientific Advances Publishers 
electronics, geophysics, meteorology and other natural and technical sciences. Reverse spectral problems today represent one of the most popular parts of the spectral analysis, which is supported by a large number of works on precisely this issue. The greatest results in the spectral theory in general and especially for inverse spectral problems has been made for Sturm-Liouville operator

$$
L[y]=-y^{\prime \prime}(x)+q(x) y,
$$

which is also called the one-dimensional Schrödinger operator. The spectral characteristics of the operator $L[y]$ imply asymptotic study of its eigenvalues, asymptotic of its intrinsic functions, decomposition in order eigenfunctions, resolve and determination tasks reverse regularized traces. A special class of problems are inverse problems for operators of the type Sturm-Liouville where the potential $q(x)$ and the argument of the function $y$ does not occur with the same argument. Differential equations that describe these operators are called differential equations with dislocated argument.

\section{Asymptotic Spectrum of the Operator $L[q, \alpha, h]$}

The border problem

$$
\begin{gathered}
-y^{\prime \prime}(x)+q(x) y(\alpha x)=\lambda y, \quad(0<\alpha<1), \\
y^{\prime}(0)-h y(0)=0 \\
y(\pi)=0 .
\end{gathered}
$$

Observers as a problem of its own value operator $L[q, \alpha, h]$. Just checked the solution of integral equations

$$
y(x, z)=\cos z x+\frac{h}{z} \sin z x+\frac{1}{z} \int_{0}^{x} q(t) \sin z(x-t) y(\alpha t) d t, \quad\left(z^{2}=\lambda\right) .
$$

It satisfies Equation (1) as a boundary condition (2). Equation (4) is solved by a method of successive approximations: 
$y(x, z)=\cos z x+\frac{h}{z} \sin z x+\frac{1}{z} \int_{0}^{x} q(t) \sin z(x-t) y(\alpha t) d t+\frac{h}{z^{2}} \int_{0}^{x} q(t) \sin z(x-t) \sin z \alpha t d t$

$$
\begin{aligned}
& +\sum_{l=2}^{\infty} \frac{1}{z^{l}} \int_{D_{l}(x, l)} Q\left(T_{l}\right) \sin z\left(x-t_{l}\right) \prod_{i=1}^{l-1} \sin z\left(\alpha t_{i}-t_{i+1}\right) \cos z \alpha t_{i} d T_{l} \\
& +h \sum_{l=2}^{\infty} \frac{1}{z^{l+1}} \int_{D_{l}(x, l)} Q\left(T_{l}\right) \sin z\left(x-t_{l}\right) \prod_{i=1}^{l-1} \sin z\left(\alpha t_{i}-t_{i+1}\right) \sin z \alpha t_{i} d T_{l} .
\end{aligned}
$$

From (5) and the boundary conditions $y(\pi)=0$, we obtain the characteristic function of the operator $L[q, \alpha, h]$ :

$$
\begin{aligned}
F(z)= & \cos \pi z+\frac{h}{z} \sin \pi z+\frac{1}{z} \int_{0}^{\pi} q(t) \sin z(\pi-t) \cos z \alpha t d t+\frac{h}{z^{2}} \int_{0}^{\pi} q(t) \sin z(\pi-t) \sin z \alpha t d t \\
& +\sum_{l=2}^{\infty} \frac{1}{z^{l}} \int_{D_{l}(x, l)} Q\left(T_{l}\right) \sin z\left(\pi-t_{l}\right) \prod_{i=1}^{l-1} \sin z\left(\alpha t_{i}-t_{i+1}\right) \cos z \alpha t_{i} d T_{l} \\
& +\sum_{l=2}^{\infty} \frac{1}{z^{l+1}} \int_{D_{l}(x, l)} Q\left(T_{l}\right) \sin z\left(\pi-t_{l}\right) \prod_{i=1}^{l-1} \sin z\left(\alpha t_{i}-t_{i+1}\right) \sin z \alpha t_{i} d T_{l} .
\end{aligned}
$$

Theorem 1. If $q(x) \in L_{1}[0, \pi]$, own values operator $L[q, \alpha, h]$ has asymptotic

$$
\lambda_{n}=\left(n+\frac{1}{2}\right)^{2}+\frac{2 h}{\pi}+O\left(\frac{1}{n+\frac{1}{2}}\right) .
$$

Proof. From rating

$$
I_{1}=\int_{0}^{\pi} q(t) \sin z(\pi-(1-\alpha) t) d t=O\left(\frac{1}{|z|}\right),
$$




$$
I_{2}=\int_{0}^{\pi} q(t) \sin z(\pi-(1+\alpha) t) d t=O\left(\frac{1}{|z|}\right)
$$

follows

$$
\int_{0}^{\pi} q(t) \sin z(\pi-t) \cos z \alpha t d t=O\left(\frac{1}{|z|}\right), \quad(z \in R, z \rightarrow \infty) .
$$

Similarly, it shows that

$$
\int_{0}^{\pi} q(t) \sin z(\pi-t) \sin z \alpha t d t=O\left(\frac{1}{|z|}\right), \quad(z \in R, z \rightarrow \infty) .
$$

It is also

$$
\begin{array}{r}
\frac{1}{z^{l}} \int_{D_{l}(\alpha)} Q\left(T_{l}\right) \sin z\left(\pi-t_{l}\right) \prod_{i=1}^{l-1} \sin z\left(\alpha t_{i}-t_{i+1}\right) \cos z \alpha t_{i} d T_{l}=O\left(\frac{1}{|z|^{l+1}}\right), \\
\frac{1}{z^{l}} \int_{D_{l}(\alpha)} Q\left(T_{l}\right) \sin z\left(\pi-t_{l}\right) \prod_{i=1}^{l-1} \sin z\left(\alpha t_{i}-t_{i+1}\right) \sin z \alpha t_{i} d T_{l}=O\left(\frac{1}{|z|^{l+2}}\right), \\
(l=2,3, \ldots) .
\end{array}
$$

From these assessments obtained asymptotic characteristic functions on the real axis:

$$
F(z)=\cos \pi z+\frac{h}{z} \sin \pi z+O\left(\frac{1}{z^{2}}\right)
$$

Let

$$
z_{n}=n^{\prime}+\frac{c_{1}}{n^{\prime}}+O\left(\frac{1}{n^{\prime 2}}\right)\left(n^{\prime}=n+\frac{1}{2}, n \in Z\right)
$$


Then, for sufficiently large modulo $n$

$$
\begin{gathered}
\cos \pi z_{n}=(-1)^{n+1} \frac{c_{1} \pi}{n^{\prime}}+O\left(\frac{1}{n^{\prime 2}}\right), \\
\sin \pi z_{n}=(-1)^{n}+O\left(\frac{1}{n^{\prime 2}}\right), \\
\frac{1}{z_{n}^{2}}=\frac{1}{n^{\prime 2}}+O\left(\frac{1}{n^{\prime 4}}\right),
\end{gathered}
$$

and

$$
F\left(z_{n}\right)=\frac{(-1)^{n}}{n^{\prime}}\left(h-c_{1} \pi\right)+O\left(\frac{1}{n^{\prime 2}}\right)
$$

If the

$$
h-c_{1} \pi=0, \quad c_{1}=\frac{h}{\pi},
$$

we get $F\left(z_{n}\right)=O\left(\frac{1}{n^{\prime 2}}\right)$

So, for $c_{1}=\frac{h}{\pi}$,

zero function $F(z)$ are determined with an accuracy up to $O\left(\frac{1}{n^{\prime 2}}\right)$. Then

$$
\lambda_{n}=z_{n}^{2}=\left(n+\frac{1}{2}\right)^{2}+\frac{2 h}{\pi}+O\left(\frac{1}{\left(n+\frac{1}{2}\right)^{2}}\right)
$$

Theorem 2. If the function $q(x)$ is absolutely continuous, for asymptotic spectrum valid

$$
\lambda_{n}=\left(n+\frac{1}{2}\right)^{2}+\frac{2 h}{\pi}+\frac{\frac{2(-1)^{n} q(\pi)}{\pi\left(1-\alpha^{2}\right)} \cos \left(n+\frac{1}{2}\right) \alpha \pi}{n+\frac{1}{2}}+O\left(\frac{1}{\left(n+\frac{1}{2}\right)^{2}}\right) .
$$


Proof. If the integrals $I_{1}$ and $I_{2}$ from the previous theorem application integration by parts, we get

$$
\begin{array}{r}
\int_{0}^{\pi} q(t) \sin z(\pi-t) \cos z \alpha t d t=\frac{1}{z}\left(\frac{q(\pi)}{1-\alpha^{2}} \cos \alpha \pi z-\frac{q(0)}{1-\alpha^{2}} \cos \pi z\right) \\
-\frac{1}{2 z}\left(\frac{1}{1-\alpha} I_{1}^{\prime}+\frac{1}{1+\alpha} I_{2}^{\prime}\right),
\end{array}
$$

where is

$$
\begin{aligned}
& I_{1}^{\prime}=\int_{0}^{\pi} q^{\prime}(t) \sin z(\pi-(1-\alpha) t) d t, \\
& I_{2}^{\prime}=\int_{0}^{\pi} q^{\prime}(t) \sin z(\pi-(1+\alpha) t) d t .
\end{aligned}
$$

From the absolute continuity of $q(x)$, it follows that $q^{\prime}(x) \in L_{1}[0, \pi]$ and, therefore, valid assessment

$$
\begin{aligned}
& I_{1}^{\prime}=O\left(\frac{1}{|z|}\right), \\
& I_{2}^{\prime}=O\left(\frac{1}{|z|}\right),
\end{aligned}
$$

is

$$
\int_{0}^{\pi} q(t) \sin z(\pi-t) \cos z \alpha t d t=\frac{1}{z}\left(\frac{q(\pi)}{1-\alpha^{2}} \cos \alpha \pi z-\frac{q(0)}{1-\alpha^{2}} \cos \pi z\right)+O\left(\frac{1}{z^{2}}\right) .
$$

Appropriate assessment of the remaining addend in the expression for $F(z)$ obtained as in the previous theorem. The characteristic function has the following asymptotic decomposition:

$$
F(z)=\cos \pi z+\frac{h}{z} \sin \pi z+\frac{1}{z^{2}}\left(\xi_{1} \cos \alpha \pi z+\xi_{2} \cos \pi z\right)+O\left(\frac{1}{z^{3}}\right),
$$


where is

$$
\xi_{1}=\frac{q(\pi)}{1-\alpha^{2}}, \quad \xi_{2}=-\frac{q(0)}{1-\alpha^{2}} .
$$

Let

$$
z_{n}=n^{\prime}+\frac{c_{1}}{n^{\prime}}+\frac{c_{2}}{n^{\prime 2}}+O\left(\frac{1}{n^{\prime 3}}\right)
$$

From the asymptotic expansions

$$
\begin{gathered}
\cos \pi z_{n}=(-1)^{n+1} \frac{c_{1} \pi}{n^{\prime}}+\frac{\pi c_{2}}{n^{\prime 2}}+O\left(\frac{1}{n^{\prime 3}}\right), \\
\sin \pi z_{n}=(-1)^{n}+O\left(\frac{1}{n^{\prime 2}}\right), \\
\cos \alpha \pi z_{n}=\cos n^{\prime} \alpha \pi+O\left(\frac{1}{n^{\prime}}\right),
\end{gathered}
$$

follow

$$
F\left(z_{n}\right)=\frac{(-1)^{n}}{n^{\prime}}\left(h-\pi c_{1}\right)+\frac{1}{n^{\prime 2}}\left[(-1)^{n+1} \pi c_{2}+\xi_{1} \cos n^{\prime} \alpha \pi\right]+O\left(\frac{1}{n^{\prime 3}}\right) .
$$

Constants $\quad c_{1}$ and $c_{2}$ determine the conditions $h-\pi c_{1}=0$ and $(-1)^{n+1} \pi c_{2}+\xi_{1} \cos n^{\prime} \alpha \pi$. We get

$$
\begin{aligned}
& c_{1}=\frac{h}{\pi}, c_{2}=\frac{(-1)^{n} q(\pi)}{\pi\left(1-\alpha^{2}\right)} \cos n^{\prime} \alpha \pi, \text { and is } \\
& \lambda_{n}=z_{n}^{2}=\left(n+\frac{1}{2}\right)^{2}+\frac{2 h}{\pi}+\frac{\frac{2(-1)^{n} q(\pi)}{\pi\left(1-\alpha^{2}\right)} \cos \left(n+\frac{1}{2}\right) \alpha \pi}{n+\frac{1}{2}}+O\left(\frac{1}{\left(n+\frac{1}{2}\right)^{2}}\right) .
\end{aligned}
$$


Theorem 3. If $q^{\prime}(x)$ is absolutely continuous function, range operators $L[q, \alpha, h]$ has an asymptotic

$$
\begin{aligned}
& \lambda_{n}=\left(n+\frac{1}{2}\right)^{2}+\frac{2 h}{\pi}+\frac{\frac{2(-1)^{n} q(\pi)}{\pi\left(1-\alpha^{2}\right)} \cos \left(n+\frac{1}{2}\right) \alpha \pi}{n+\frac{1}{2}} \\
& +\frac{\frac{h^{2}}{\pi^{2}}-\frac{2 h^{3}}{3 \pi}+\frac{2 h q(0)}{\pi(1+\alpha)}-\frac{2\left(1+\alpha^{2}\right) q^{\prime}(0)}{\left(1-\alpha^{2}\right)^{2}}+\frac{2(-1)^{n}}{\pi}\left(\frac{h q(\pi)}{1+\alpha}+\frac{2 \alpha q^{\prime}(\pi)}{\left(1-\alpha^{2}\right)^{2}}\right) \sin \left(n+\frac{1}{2}\right) \alpha \pi}{\left(n+\frac{1}{2}\right)^{2}} \\
& +O\left(\frac{1}{\left(n+\frac{1}{2}\right)^{3}}\right) .
\end{aligned}
$$

Proof. In this case, the $q^{\prime \prime}(x) \in L_{1}[0, \pi]$ then apply marks

$$
\begin{aligned}
& I_{1}^{\prime}=-\frac{q^{\prime}(\pi)}{z(1-\alpha)} \sin \alpha \pi z+\frac{q^{\prime}(0)}{z(1-\alpha)} \sin \pi z+O\left(\frac{1}{z^{2}}\right), \\
& I_{2}^{\prime}=-\frac{q^{\prime}(\pi)}{z(1+\alpha)} \sin \alpha \pi z+\frac{q^{\prime}(0)}{z(1+\alpha)} \sin \pi z+O\left(\frac{1}{z^{2}}\right),
\end{aligned}
$$

and, based on them, rating

$$
\begin{aligned}
& \int_{0}^{\pi} q(t) \sin z(\pi-t) \cos z \alpha t d t=\frac{1}{z}\left(\xi_{1} \cos \alpha \pi z+\xi_{2} \cos \pi z\right) \\
& +\frac{1}{z^{2}}\left[\frac{2 \alpha q^{\prime}(\pi)}{\left(1-\alpha^{2}\right)^{2}} \sin \alpha \pi z-\frac{\left(1+\alpha^{2}\right) q^{\prime}(0)}{\left(1-\alpha^{2}\right)^{2}} \sin \pi z\right]+O\left(\frac{1}{z^{3}}\right)
\end{aligned}
$$

Similarly, it shows that

$$
\int_{0}^{\pi} q(t) \sin z(\pi-t) \sin z \alpha t d t=\frac{1}{z}\left(\frac{q(\pi)}{1-\alpha^{2}} \sin \alpha \pi z-\frac{q(0)}{1-\alpha^{2}} \sin \pi z\right)+O\left(\frac{1}{z^{2}}\right) .
$$


Other summands in the expression for $F(z)$ are in order $O\left(\frac{1}{z^{4}}\right)$, and is

$$
\begin{aligned}
F(z)=\cos \pi z+ & \frac{h}{z} \sin \pi z+\frac{1}{z^{2}}\left(\xi_{1} \cos \alpha \pi z+\xi_{2} \cos \pi z\right) \\
& +\frac{1}{z^{3}}\left(\xi_{3} \sin \alpha \pi z+\xi_{4} \sin \pi z\right)+O\left(\frac{1}{z^{4}}\right), \quad(z \in R, z \rightarrow \infty),
\end{aligned}
$$

where is

$$
\xi_{3}=\frac{h q(\pi)}{1-\alpha^{2}}+\frac{2 \alpha q^{\prime}(\pi)}{\left(1-\alpha^{2}\right)^{2}}, \quad \xi_{4}=-\frac{\alpha h q(0)}{1-\alpha^{2}}-\frac{\left(1+\alpha^{2}\right) q^{\prime}(0)}{\left(1-\alpha^{2}\right)^{2}} .
$$

If is $z_{n}=n^{\prime}+\frac{c_{1}}{n^{\prime}}+\frac{c_{2}}{n^{\prime 2}}+\frac{c_{3}}{n^{\prime 3}}+O\left(\frac{1}{n^{\prime 4}}\right)$, then from the asymptotic expansions

$$
\begin{gathered}
\cos \pi z_{n}=(-1)^{n+1}\left(\frac{c_{1} \pi}{n^{\prime}}+\frac{\pi c_{2}}{n^{\prime 2}}+\frac{\pi c_{3}-\frac{\pi^{3} c_{1}^{3}}{6}}{n^{\prime 3}}\right)+O\left(\frac{1}{n^{\prime 4}}\right) \\
\sin \pi z_{n}=(-1)^{n}\left(1-\frac{\frac{\pi^{2} c_{1}^{2}}{2}}{n^{\prime 2}}\right)+O\left(\frac{1}{n^{\prime 3}}\right) \\
\cos \alpha \pi z_{n}=\cos n^{\prime} \alpha \pi-\frac{\alpha \pi c_{1}}{n^{\prime}}+O\left(\frac{1}{n^{\prime 2}}\right) \\
\sin \alpha \pi z_{n}=\sin n^{\prime} \alpha \pi+O\left(\frac{1}{n^{\prime}}\right)
\end{gathered}
$$


follow

$$
\begin{aligned}
F\left(z_{n}\right)= & \frac{(-1)^{n}}{n^{\prime}}\left(h-\pi c_{1}\right)+\frac{1}{n^{\prime 2}}\left[(-1)^{n+1} \pi c_{2}+\xi_{1} \cos n^{\prime} \alpha \pi\right] \\
& +\frac{1}{n^{\prime 3}}\left[(-1)^{n+1} \pi c_{3}-\frac{\pi^{3} c_{1}^{3}}{6}+(-1)^{n+1} \frac{\pi^{2} c_{1}^{2} h}{2}+(-1)^{n+1} \xi_{2} \pi c_{1}\right. \\
& \left.+\xi_{3} \sin n^{\prime} \alpha \pi+(-1)^{n} \xi_{4}\right]+O\left(\frac{1}{n^{\prime 4}}\right) .
\end{aligned}
$$

Equating the expression in brackets to zero, we obtain the $c_{1}=\frac{h}{\pi}$, $c_{2}=\frac{(-1)^{n} q(\pi)}{\pi\left(1-\alpha^{2}\right)} \cos n^{\prime} \alpha \pi$, and $c_{3}=-\frac{h^{3}}{3 \pi}+\frac{h q(0)}{\pi(1+\alpha)}-\frac{\left(1+\alpha^{2}\right) q^{\prime}(0)}{\pi\left(1-\alpha^{2}\right)^{2}}+\frac{(-1)^{n}}{\pi}\left(\frac{h q(\pi)}{(1+\alpha)}+\frac{2 \alpha q^{\prime} \pi}{\left(1-\alpha^{2}\right)^{2}}\right) \sin n^{\prime} \alpha \pi$.

Substituting $c_{1}, c_{2}$, and $c_{3}$ in the expression $\lambda_{n}=n^{\prime 2}+2 c_{1}+\frac{2 c_{2}}{n}$ $+\frac{c_{1}^{2}+2 c_{3}}{n^{\prime 2}}+O\left(\frac{1}{n^{\prime 3}}\right)$ we get specified asymptotic.

\section{Asymptotic Spectrum of the Operator $L[q, \alpha, h, H]$}

The operator $L[q, \alpha, h, H]$ is defined by the border problem with general boundary condition:

$$
\begin{gathered}
-y^{\prime \prime}(x)+q(x) y(\alpha x)=\lambda y(x), \quad(0<\alpha<1), \\
y^{\prime}(0)-h y(0)=0, \\
y^{\prime}(\pi)+H y(\pi)=0,
\end{gathered}
$$

where are $h, H \in C$. 
Method of variation constants shows that the Equation (6) with the boundary condition (7) equivalent integral equation

$$
y(x, z)=\cos z x+\frac{h}{z} \sin z x+\frac{1}{z} \int_{0}^{x} q(t) \sin z(x-t) y(\alpha t) d t,\left(z^{2}=\lambda\right) .
$$

The solution of the Equation (9) is

$$
\begin{aligned}
y(x, z)= & \cos z x+\frac{h}{z} \sin z x+\frac{1}{z} \int_{0}^{x} q(t) \sin z(x-t) \cos z \alpha t d t+\frac{h}{z^{2}} \int_{0}^{x} q(t) \sin z(x-t) \sin z \alpha t d t \\
& +\sum_{l=2}^{\infty} \frac{1}{z^{l}} \int_{D_{l}(x, l)} Q\left(T_{l}\right) \sin z\left(x-t_{l}\right) \prod_{i=1}^{l-1} \sin z\left(\alpha t_{i}-t_{i+1}\right) \cos z \alpha t_{i} d T_{l} \\
& +\sum_{l=2}^{\infty} \frac{1}{z^{l+1}} \int_{D_{l}(x, l)} Q\left(T_{l}\right) \sin z\left(x-t_{l}\right) \prod_{i=1}^{l-1} \sin z\left(\alpha t_{i}-t_{i+1}\right) \sin z \alpha t_{i} d T_{l} .
\end{aligned}
$$

Boundary condition (8) determines the characteristic function of the operator $L[q, \alpha, h, H]$ :

$$
\begin{gathered}
F(z)=-z \sin \pi z+(h+H) \cos \pi z+\int_{0}^{\pi} q(t) \cos z(\pi-t) \cos z \alpha t d t+\frac{1}{z} \\
{\left[h H \sin \pi z+h \int_{0}^{\pi} q(t) \cos z(\pi-t) \sin z \alpha t d t+H \int_{0}^{\pi} q(t) \sin z(\pi-t) \cos z \alpha t d t\right]} \\
+\frac{h H}{z^{2}} \int_{0}^{\pi} q(t) \sin z(\pi-t) \sin z \alpha t d t \\
+\sum_{l=2}^{\infty} \frac{1}{z^{l-1}} \int_{D_{l}(\alpha)} Q\left(T_{l}\right) \cos z\left(\pi-t_{l}\right) \prod_{i=1}^{l-1} \sin z\left(\alpha t_{i}-t_{i+1}\right) \cos z \alpha t_{i} d T_{l} \\
+h \sum_{l=2}^{\infty} \frac{1}{z^{l}} \int_{D_{l}(\alpha)} Q\left(T_{l}\right) \cos z\left(\pi-t_{l}\right) \prod_{i=1}^{l-1} \sin z\left(\alpha t_{i}-t_{i+1}\right) \sin z \alpha t_{i} d T_{l}
\end{gathered}
$$




$$
\begin{aligned}
& +H \sum_{l=2}^{\infty} \frac{1}{z^{l}} \int_{D_{l}(\alpha)} Q\left(T_{l}\right) \sin z\left(\pi-t_{l}\right) \prod_{i=1}^{l-1} \sin z\left(\alpha t_{i}-t_{i+1}\right) \cos z \alpha t_{i} d T_{l} \\
& +h H \sum_{l=2}^{\infty} \frac{1}{z^{l+1}} \int_{D_{l}(\alpha)} Q\left(T_{l}\right) \sin z\left(\pi-t_{l}\right) \prod_{i=1}^{l-1} \sin z\left(\alpha t_{i}-t_{i+1}\right) \sin z \alpha t_{i} d T_{l} .
\end{aligned}
$$

For asymptotic apply the following theorem:

Theorem 4. If it is $q(x) \in L_{1}[0, \pi]$, then

$$
\begin{aligned}
& F(z)=z \cos \pi z+H \sin \pi z+O\left(\frac{1}{z}\right) \\
& \lambda_{n}=\left(n+\frac{1}{2}\right)^{2}+\frac{2 H}{\pi}+O\left(\frac{1}{n+\frac{1}{2}}\right)
\end{aligned}
$$

Theorem 5. If $q(x)$ is absolutely continuous function, then

$$
\begin{gathered}
F(z)=z \cos \pi z+H \sin \pi z+\frac{1}{z}\left(\xi_{1} \cos \alpha \pi z+\xi_{2} \cos \pi z\right)+O\left(\frac{1}{z^{2}}\right) \\
\xi_{1}=\frac{q(\pi)}{1-\alpha^{2}}, \quad \xi_{2}=-\frac{q(0)}{1-\alpha^{2}} \\
\lambda_{n}=\left(n+\frac{1}{2}\right)^{2}+\frac{2 H}{\pi}+\frac{\frac{2(-1)^{n} \alpha q(\pi)}{\pi\left(1-\alpha^{2}\right)} \cos \left(n+\frac{1}{2}\right) \alpha \pi}{n+\frac{1}{2}}+O\left(\frac{1}{\left(n+\frac{1}{2}\right)^{2}}\right) .
\end{gathered}
$$

Theorem 6. If $q(x)$ is absolutely continuous function, then says the following ratings:

$$
\begin{aligned}
F(z)=z \cos \pi z & +H \sin \pi z+\frac{1}{z}\left(\xi_{1} \cos \alpha \pi z+\xi_{2} \cos \pi z\right) \\
& +\frac{1}{z^{2}}\left(\xi_{3} \sin \alpha \pi z+\xi_{4} \sin \pi z\right)+O\left(\frac{1}{z^{3}}\right)
\end{aligned}
$$




$$
\begin{gathered}
\xi_{3}=\frac{H q(\pi)}{1-\alpha^{2}}+\frac{\left(1+\alpha^{2}\right) \alpha q^{\prime}(\pi)}{\left(1-\alpha^{2}\right)^{2}}, \quad \xi_{4}=-\frac{\alpha H q(0)}{1-\alpha^{2}}-\frac{2 \alpha q^{\prime}(0)}{\left(1-\alpha^{2}\right)^{2}}, \\
\lambda_{n}=\left(n+\frac{1}{2}\right)^{2}+\frac{2 H}{\pi}+\frac{\frac{2(-1)^{n} q(\pi)}{\pi\left(1-\alpha^{2}\right)} \cos \left(n+\frac{1}{2}\right) \alpha \pi}{n+\frac{1}{2}} \\
+\frac{-\frac{2 H^{3}}{3 \pi}-\frac{4 \alpha q^{\prime}(0)}{\pi\left(1-\alpha^{2}\right)^{2}}+\frac{2(-1)^{n}}{\pi}\left[H q(\pi)+\left(1+\alpha^{2}\right) q^{\prime}(\pi)\right] \sin \left(n+\frac{1}{2}\right) \alpha \pi}{\left(n+\frac{1}{2}\right)^{2}} \\
+O\left(\frac{1}{\left(n+\frac{1}{2}\right)^{3}}\right) .
\end{gathered}
$$

The evidence of this proposition does not substantially differ from the evidence of appropriate proposition for the operator $L[q, \alpha, h]$, and were thus excluded.

\section{Conclusion}

This work is devoted to determining the spectral characteristics of some types of operators Sturm-Liouville delay. The results in this paper are supported in various fields of mathematical analysis, such as: The theory of regularized traces of differential operators and the theory of analytical functions. These results are a good basis for solving inverse problems operator are Sturm-Liouville delay method of Fourier coefficients, which is a new method to this problem and applied a few years ago. 


\section{References}

[1] G. Freiling and V. Yurko, Inverse Sturm-Liouville Problems and their Applications, Nova Science Publishers, Inc. Huntington, New York, 2008.

[2] V. Ambarzumjan, Uber eine Frage der Eigenwerttheorie, Zeitshr. für Physik 53 (1929), 690-695.

[3] I. Kalčo, Construction solutions differential operator type Sturm-Liouville border task with linear delay, Bulletin of the international mathematical virtual institute ISSN (p) 2303-4874, ISSN (o) 2303-4955, 0.951.

[4] Göran Borg, Eine Umkehrung der Sturm-Liouvilleschen Eigenwertaufgabe, Acta Math. 78 (1946), 1-96.

[5] N. Levinson, The inverse Sturm-Liouville problem, Math. Tidssker. 13 (1949), 25-30.

[6] R. Carlson, Inverse spectral theory for some singular Sturm-Liouville problems, J. Diff. Equations 106 (1993), 121-140.

[7] G. Freiling and V. Yurko, Inverse problems for Sturm-Liouville differential operators with a constant delay, Applied Mathematical Letters (2012). 